Approved for public release;

distribution is unlimited

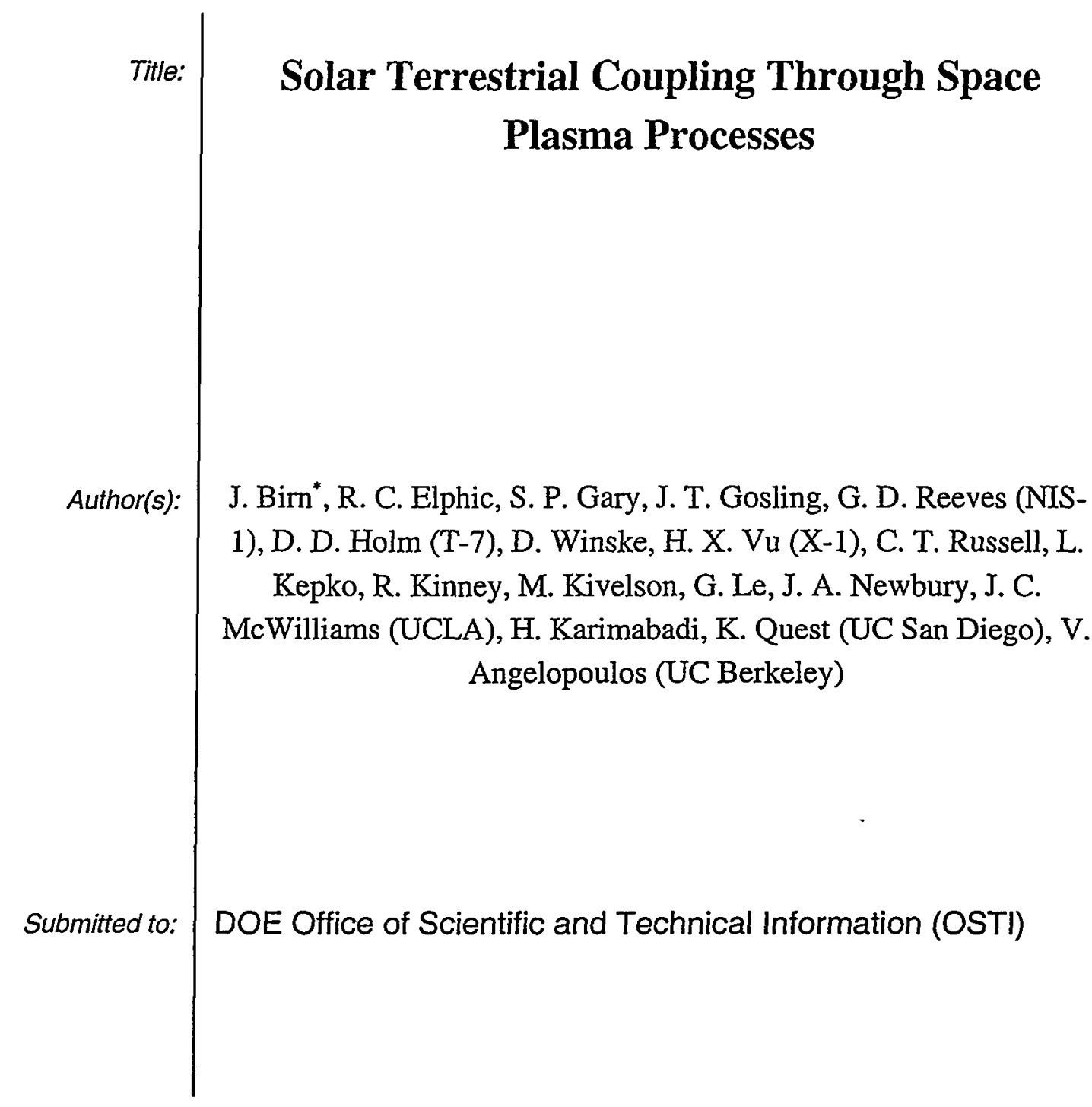

Los Alamos National Laboratory, an affirmative action/equal opportunity employer, is operated by the University of California for the U.S. Department of Energy under contract W-7405-ENG-36. By acceptance of this article, the publisher recognizes that the U.S. Government retains a nonexclusive, royaltyfree license to publish or reproduce the published form of this contribution, or to allow others to do so, for U.S. Government purposes. Los Alamos National Laboratory requests that the publisher identify this article as work performed under the auspices of the U.S. Department of Energy. Los Alamos National Laboratory strongly supports academic freedom and a researcher's right to publish; as an institution, however, the Laboratory does not endorse the viewpoint of a publication or guarantee its technical correctness. 


\section{DISCLAIMER}

This report was prepared as an account of work sponsored by an agency of the United States Government. Neither the United States Government nor any agency thereof, nor any of their employees, make any warranty, express or implied, or assumes any legal liability or responsibility for the accuracy, completeness, or usefulness of any information, apparatus, product, or process disclosed, or represents that its use would not infringe privately owned rights. Reference herein to any specific commercial product, process, or service by trade name, trademark, manufacturer, or otherwise does not necessarily constitute or imply its endorsement, recommendation, or favoring by the United States Government or any agency thereof. The views and opinions of authors expressed herein do not necessarily state or reflect those of the United States Government or any agency thereof. 


\section{DISCLAIMER}

Portions of this document may be illegible in electronic image products. Images are produced from the best available original document. 


\title{
Solar Terrestrial Coupling Through Space Plasma Processes
} 97803

J. Birn*, R. C. Elphic, S. P. Gary, J. T. Gosling, G. D. Reeves (NIS-1), D. D. Holm (T-7), D. Winske, H. X. Vu (X-1), C. T. Russell, L. Kepko, R. Kinney, M. Kivelson, G. Le, J. A. Newbury, J. C. McWilliams (UCLA), H. Karimabadi, K. Quest (UC San Diego), V. Angelopoulos (UC Berkeley)

\begin{abstract}
This is the final report of a three-year, Laboratory-Directed Research and Development (LDRD) project at the Los Alamos National Laboratory (LANL). The project investigates plasma processes that govern the interaction between the solar wind, charged particles ejected from the sun, and the earth's magnetosphere, the region above the ionosphere governed by the terrestrial magnetic field. Primary regions of interest are the regions where different plasma populations interact with each other. These are regions of particularly dynamic plasma behavior, associated with magnetic flux and energy transfer and dynamic energy release. The investigations concerned charged particle transport and energization, and microscopic and macroscopic instabilities in the magnetosphere and adjacent regions. The approaches combined space data analysis with theory and computer simulations.
\end{abstract}

\section{Background and Research Objectives}

The earth's magnetosphere is defined as its charged particle (plasma) environment above the ionosphere governed by the terrestrial magnetic field. The magnetosphere is shaped and strongly controlled by its interaction with the solar wind, a charged particle stream ejected from the sun. The magnetosphere occupies a region of roughly 10 to 15 earth radii above the surface on the dayside and extends into a long tail, like a comet's tail, of hundreds of earth radii length on the nightside. The major regions are shown schematically in Fig. 1. Dynamic processes that involve the coupling between the two plasmas cause the spectacular auroras (polar lights),

\footnotetext{
*Principal Investigator, e-mail: jbirn@lanl.gov
}

RECEIVEO

DEC 132000 
disturb the earth's magnetic field and long-range power lines, and affect satellite measurements and operations. The interest in magnetospheric structure and dynamics, its charged particle population, and the dynamic processes affecting the particles stems also from the fact that the underlying processes are characteristic of plasma behavior in many other areas, laboratory plasma confinement experiments as well as solar flares and astrophysical phenomena.

The magnetosphere and the solar wind are accessible, through satellites, to direct measurements of their magnetic and electric fields and particle populations. The satellites carrying the scientific instruments are tiny in comparison to the characteristic dimensions of the particle and field structures within these regions. The measurements are therefore far less disturbing and more detailed than those obtained in laboratory experiments. The detailed knowledge of particle distributions and fields in the magnetosphere and the solar wind, which may vary on a wide range of temporal and spatial scales, hence provides an ideal test ground for theoretical studies and high performance computer modeling of plasma behavior.

Primary regions of interest are the regions where one plasma population interacts with a different adjacent population or with some different surrounding medium. These are the regions of particularly dynamic plasma behavior, where plasma and energy transport and dynamic energy release occur. Key problems are the question of how this interaction takes place, what waves, instabilities, and particle/field interactions are involved, how the penetration of plasma and energy through characteristic boundaries takes place, and how the characteristic properties of the plasmas and fields of the different populations influence each other on different spatial and temporal scales. The scientific applications include the properties of the solar wind, wave, and particle interactions at the bow shock of the Earth's magnetosphere and at the magnetopause, which separates the magnetosphere from the solar wind, the influence of the solar wind 
on the overall magnetospheric structure, and the details of plasma, magnetic flux, and energy transport within the magnetospheric tail.

\section{Importance to LANL's Science and Technology Base and National R\&D Needs}

The investigations used two outstanding Laboratory capabilities. The first capability is the Laboratory's unique expertise from its thirty-year program for the development, flight, and analysis of data from instruments to measure plasma and energetic charged particles in near-Earth space. The second one is the unique Laboratory capability in high performance computing and the development and application of plasma simulation codes. An important aspect of the research was not only the application of these unique capabilities, but also the further enhancement of the capabilites through the connections with university expertise and resources. The improvement and continuing development of the plasma simulation codes is an obvious part of the interactive application of the codes to the space plasma transport and wave-particle interaction problems. The experimental and data analysis part of the proposed research, however, also enhanced the Laboratory's expertise, not only in improving the handling and analyzing of existing data sets, but also in giving strong impulses to the further development of instrumentation of future space experiments.

\section{Scientific Approach and Accomplishments}

An advanced understanding of the plasma processes involved in solar-terrestrial interactions requires a coordinated research effort in data interpretation and computer modeling. Characteristic events in the solar wind, at the magnetopause, and inside the geomagnetic tail were analyzed in detail, utilizing plasma data obtained by Los Alamos instruments and magnetic field data from UC instruments on satellites. The data analysis focused on the temporal variations of the plasma flow and the fluctuations of the 
magnetic field and their interrelation. This analysis gave new insights into the energy propagation and the dissipation processes operating in characteristic interaction regions. The distribution functions for ions and electrons provided information about the sources of the particles, anisotropies, scattering mechanisms, and the generation of high energy subpopulations, which may be crucial for dissipation processes.

The data analysis was accompanied by theoretical investigations and, in particular, computer modeling of the charged particle behavior, using data analysis results to define initial scenarios for the simulations and to select the most promising approaches. A magnetohydrodynamic (MHD) approach was used for the large-scale behavior, which governs the overall transport, the force balance between adjacent plasma regions, and the global energy and particle balance. The microscopic physics was described by kinetic approaches, using Vlasov theory and particle codes. The detailed achievements are discussed below.

\section{Solar Wind Properties.}

There are unexpected bounds to electron and proton temperatures in the ambient solar wind: there is an apparent minimum in the electron to proton temperature ratio and there is a minimum in electron beta (ratio of plasma pressure over magnetic pressure). ${ }^{6}$ These bounds are observed to vary with solar wind bulk speed and with radial distance in the heliosphere. Observational periods where these temperature bounds are observed are also coincident with enhanced electromagnetic wave activity in the $18-100 \mathrm{~Hz}$ frequency range. Initial studies of the ambient solar wind showed that the electron temperature is approximately twice the proton temperature at $1 \mathrm{AU}$ for typical solar wind velocities, but

at high solar wind velocities the two became nearly equal. ${ }^{[1]} \mathrm{A}$ later statistical study by Feldman et al. ${ }^{[2]}$ showed that this correlation arose due to the correlation between proton temperature and solar wind bulk velocity while the electron temperature remains nearly 
constant. In fact, the best "educated guess" one can make for the temperature of solar wind electrons at $1 \mathrm{AU}$ is a constant value of $140,000 \mathrm{~K}$, regardless of the other conditions in the solar wind. ${ }^{23}$ This temperature-coupling phenomenon is especially puzzling since the most common proton instabilities in the solar wind are non-resonant with electrons, and thus are independent of electron temperature. ${ }^{[3]}$ Using collisionless Vlasov theory, ${ }^{[4]}$ we have found a critical condition on the electron beta due to the competition between and subsequent heating by heat flux instabilities. ${ }^{5,6}$ Fair agreement was found between our preliminary estimates of this critical condition and observations from the spacecraft Ulysses. This result sheds new light on the basic physical control mechanisms that govern the temperature of the solar wind. ${ }^{24}$

\section{Coronal Mass Ejections}

Los Alamos research has long played a dominant role in discovering, identifying, and analyzing huge plasma clouds ejected from the solar corona, named coronal mass ejections (CMEs). CME-driven solar wind disturbances are known to be the prime cause of large, transient geomagnetic storms and major solar energetic particle events near the earth. It is well known that the size of a geomagnetic storm is directly related to the solar wind flow speed and the strength and orientation of the interplanetary magnetic field associated with a CME-driven disturbance. An effective storm forecasting system thus requires a means of determining at least these properties for earthward-directed CME events. The current study used the combination of coronagraph data obtained from nearEarth orbit and solar wind data obtained by satellites $90^{\circ}$ off the Sun-Earth line. CMEs that occurred when the satellites were above the solar limb as observed from Earth were used to determine how well we can predict speed and field enhancements in solar wind disturbances from coronagraph observations of the CMEs causing the disturbances. 
Several particularly well-defined cases of paired CME and interplanetary disturbance observations were identified and analyzed. The results of this survey showed how the disturbance speed near $1 \mathrm{AU}$ relates to the CME speed observed in the coronagraph images. Slow CMEs get accelerated up to at least the minimum speed of the normal solar wind, while fast CMEs decelerate as they interact with the ambient plasma ahead and behind. Our survey clearly showed that the fastest CMEs in the corona produced the strongest enhancements in the interplanetary magnetic field, as expected from both previous observational studies and theoretical predictions.

\section{Simulation of the Bow Shock and Magnetopause}

The magnetopause is the interface between the magnetosphere and the solar wind plasma in the magnetosheath, which has passed through the bow shock surrounding the magnetosphere (Figure 1). Its physics is controlled by both local and non-local phenomena. ${ }^{19}$ The objective of this study was to use 2-D hybrid (fluid electrons, particle ions) simulations to investigate the role of these local and non-local processes at the bow shock and the magnetopause. Fig. 2 illustrates the structure of the bow shock obtained in the simulation, as seen by the magnitude of the $y$-component of the magnetic field $\left(B_{y}\right)$ as a function of $x$ and $y$. Solar wind plasma is injected from the left boundary and is partially reflected from a finite sized obstacle placed at the center of the right hand boundary. ${ }^{25}$ The magnetic field (IMF) lies in the $x, y$ plane and for this particular example makes a $135^{\circ}$ angle with the $x$ axis. The interaction between the solar wind and the non-magnetic obstacle forms a curved bow shock. The structure of the shock strongly depends on whether its normal is perpendicular to or parallel with the upstream solar wind magnetic field. As can be seen, the bottom portion of the bow shock (corresponding to quasiperpendicular) is a sharp boundary while the top part (quasi-parallel side) has a more turbulent structure. In addition, presence of waves upstream of the quasi-parallel side of 
the bow shock is also evident. This overall structure of the shock is consistent with expectations based on spacecraft observations and localized hybrid simulations of the shock.

To illustrate the fact that the shock physics is properly captured in this simulation, Fig. 3 shows the profile of $x$ and $y$ components of the magnetic field $\left(B_{x, y}\right)$, density $(N)$, ion temperature $(\mathcal{T})$, flow velocity along the $x$ direction $\left(V_{x}\right)$, and the total magnetic field (B) along the white line shown in Fig. 2. As can be seen, a sharp change in all plasma parameters occurs at the shock with characteristics similar to those seen in localized 1-D simulations and spacecraft observations. The large-scale hybrid simulations of a curved bow shock hence reproduce the microphysics of collisionless shocks with characteristics similar to those observed by spacecraft, ${ }^{20,25}$ as well as the macroscopic shape of the shock.

We have also examined more localized physical processes associated with the magnetopause boundary. The Rayleigh-Taylor instability occurs in a magnetized plasma when a dense plasma is supported against a lighter plasma by a pseudo-gravity and a magnetic field, as occurs at the magnetopause. We have used two-dimensional hybrid simulations to study the behavior of the instability in both the long wavelength fluid regime as well as at shorter wavelengths, where ion kinetic effects become more important. We have shown that there are three distinct regimes (the fluid regime, a regime where the Hall term contributes significantly, and a third regime where unmagnetized ion effects are important), with different linear and nonlinear properties. Moreover, we have studied how nonconstant gravity affects the development of the instability (as would occur at the magnetopause due to the variability of the solar wind)..$^{27,28}$ 


\section{Tearing Instability at the Magnetopause}

A key feature of the coupling between different plasma populations across magnetic boundaries is the process of magnetic reconnection, initiated by the "tearing" instability, which leads to a magnetic connection across the boundary. ${ }^{1-4,7-9,26}$ Having established that a collisionless tearing mode is a viable candidate for the onset of reconnection at the dayside magnetosphere, we started to investigate its large-scale consequences, using a plane current sheet and "mass loaded" ions as a starting equilibrium. Given that thin current sheets are observed both at the magnetotail and the magnetopause, our plan was to study their three-dimensional stability. In addition to current carriers, we have also introduced a secondary population of ions. At the magnetopause, there are several sources of these secondary ions: cold ionospheric ions, reflected ions, accelerated flows resulting from the reconnection, and heavier elements. At the magnetotail, the secondary ions can be heavy elements such as oxygen or ion beams. We then find that both configurations are unstable to the Kelvin-Helmholtz instability. This instability is quite robust and grows to large amplitudes (Figure 4). The usual Kelvin-Helmholtz instability is thought to occur away from the subsolar point, where there exists a velocity shear due to convection. However, our finding suggests that the instability can occur both near and away from the subsolar point wherever exists a thin current layer.

In order to get a handle on the instability as well as the need to perform a parameter study to determine the conditions under which the Kelvin-Helmholtz instability can have sufficient growth to play a significant role at the magnetopause, we developed a fluid linear theory of the instability. We have been able to carry out our theory for a general plasma and field configuration. This theory is useful in that we were able to pin down the nature of the instability that we were seeing in our simulations. 


\section{Magnetopause Flux Transfer Events.}

The magnetopause is frequently characterized by transient perturbations of the magnetic field, called "flux transfer events" (FTEs), which have been interpreted to be manifestations of reconnection between the magnetospheric and magnetosheath magnetic fields. ${ }^{[11,14]}$ Plasma signatures of FTEs are relatively poorly understood. In the plasma moment data (density, flow speed, temperature and heat flux), only a subset of flux transfer events exhibit signatures that distinguish them from adjacent magnetosheath or magnetospheric plasma. For an FTE in the magnetosheath, the observed plasma signatures include a decrease in density, an increase in temperature and heat flux, and sometimes an enhancement of flow speed..$^{[5-10]}$ Such plasma signatures suggest that the plasma within an FTE is a mixture of magnetosheath and magnetospheric plasma. Thomsen et al. ${ }^{[12]}$ studied the plasma velocity distribution functions within FTEs and confirmed that the plasma within both magnetosheath and magnetospheric FTEs indeed consists of a mixture of magnetosheath and magnetospheric populations, as suggested from plasma moment data.

The objective of this effort was to determine the source of plasmas within FTEs and to learn how FTEs are formed. We determined the interior structure of flux transfer events by examining high-resolution magnetic field and plasma distribution functions from the ISEE 2 spacecraft. Based on prior studies, we have confirmed the existence of two distinct regions within an FTE: a central core and a field draping region; and these two regions have been found within FTEs observed both on the magnetosheath side and the magnetospheric side of the magnetopause. The boundaries between the two regions are apparent in both the field and the plasma data. The plasma signatures within FTEs show a mixture of magnetospheric and magnetosheath plasmas (Fig. 5) and thus prove the connection of the interplanetary magnetic field and the Earth's magnetic field. 
They indicate that the magnetic field lines within the central core region are open allowing the inflow of cold magnetosheath plasma and the outflow of hot magnetospheric plasma through the open flux tube. The reconnection picture is further supported by the observation of separate electron and ion edges at the trailing boundary of a northward moving flux tube, expected as time-of-flight effects on newly reconnected field lines. Thus, our observations are consistent with the reconnected open flux tube interpretation for FTEs. ${ }^{20,21}$

The data studies were complemented by two-dimensional and three-dimensional particle simulation studies. ${ }^{12-14,19,25}$ These studies have shown that non-local effects play a critical role in the formation of the discontinuities in the magnetopause transition layer. This in turn leads to structures that in most cases do not have one-dimensional counterparts, and attempts to classify them as such would lead to erroneous identification. This calls into question previous studies based on one-dimensional simulations but is consistent with the observational fact that during most magnetopause crossings, it is hard to identify a "clean" rotational discontinuity $(\mathrm{RD})$. Further, the previously held view that rotations of $180^{\circ}$ at the magnetopause are intermediate shocks whereas other rotational angles would be RDs is also questionable. In a related study, we showed that in 3-D, ion physics can lead to intermittent reconnection. This finding was quite unexpected and is contrary to conventional wisdom. We have established that there exists a critical value of resistivity below which reconnection becomes intermittent. The relevance of this intermittent reconnection mechanism to the size and rate of occurrence of flux transfer events (FTEs) ${ }^{[14]}$ is currently under investigation.

\section{Bursty Flows and Plasmoids in the Magnetotail.}

Two phenomena observed in the magnetotail suggest that transient reconnection akin to dayside flux transfer events also occur in the magnetotail. 
These are brief bursts of fast plasma flow ("bursty bulk flows," BBFs ${ }^{[15]}$ ) and plasmoids or magnetic flux ropes. The latter consist of magnetic field line structures which represent closed loops or helical field lines within the tail plasma sheet (Fig. 1). Magnetic flux ropes are distinguished from plasmoids by the presence of a strong magnetic field (core field) in the center of the helical structure. ${ }^{10,11,17}$ Both types of events exhibit episodic behavior, illustrated in Figure 6, showing the magnetic field data from two closely spaced spacecraft ISEE 1 and 2, and the trajectory through the structure. This event consisted of rapid thinning of the plasma sheet, followed by $1000 \mathrm{~km} / \mathrm{sec}$ tailward flow. Near the end of the flow ISEE1 and 2 simultaneously observed two different flux ropes, with different core field strengths. This implies that at least $3 \mathrm{x}$-lines were active during the event. Our multipoint observations proved a direct link between BBFs and flux ropes. Furthermore, we have also established a direct link between BBFs and characteristic magnetic pulsations observed on the ground.

If $\mathrm{BBFs}$ are caused by azimuthally limited, transient reconnection on closed plasma sheet field lines, then each BBF should create an azimuthally limited flux rope. If the reconnection has not connected lobe flux, then this flux rope will be "fossilized," i.e., contained within the plasma sheet. Using constraints from the two-spacecraft observations, we have started modeling the flux ropes. The goal was to determine if these near-Earth flux ropes are force-free (that is, governed by the absence of magnetic forces), to investigate the fate of "fossilized" flux ropes, and to examine the question of core-field formation.

We found that most of the events do not fit a model based on force balance. ${ }^{[13]}$ This indicates an important role of time dependence, anisotropic stresses, or dynamic pressure from the outflow of a reconnection site, pushing the flux rope downtail, or the pile up of magnetic flux at the leading edge. Figure 7 shows a flux rope that is clearly not in force balance, as evident by the difference in the $\mathrm{B}_{\mathrm{z}}$ component of the field on either 
side of the flux rope. Note the strong compression of the trailing edge in $B_{z}$. The continued flow after the flux rope has passed suggests that the $\mathrm{x}$-line is still active, and is pushing the flux rope downtail. Our results further suggest that a substantial fraction of the core-field forms during reconnection and that the local conditions played an important role in its formation.

In an additional theoretical study, ${ }^{14}$ we examined the generation of core fields within flux ropes in a simulation that included Hall currents. We showed that the Hall term plays a critical role in determining the magnetic structure of these flux ropes and demonstrated how large core fields naturally arise in flux ropes due to Hall currents, without having to invoke external mechanisms.

\section{Turbulent Space and Astrophysical Plasmas}

Natural plasmas are often found with a strong large-scale magnetic field. A background field will support linear waves and the nonlinear interaction of these waves is the source of many interesting phenomena. Strong nonlinear interaction results in turbulence, and the background field can have a strong influence even on scales much smaller than that of the field.

Strong anisotropy of density fluctuations in the interstellar medium are correlated with the galactic magnetic field. In the solar wind, the interplanetary magnetic field causes elliptical distortions of distant objects. Direct spacecraft measurements of fluctuating fields in the solar wind show a strong suppression of fluctuations along the interplanetary magnetic field compared with the perpendicular direction. The source of fluctuating fields on the sun is presumably isotropic, so the development of anisotropy is a fundamental question of magnetic turbulence, which has still not been fully explained.

Linear waves and their nonlinear interaction are also observable in the near-earth space environment. 
Closed magnetic field lines support Alfvén waves, which are easily observed from the ground and in space. The waves are partially reflected either at the ionosphere or at the auroral acceleration region at 1-2 earth radii. During magnetic substorms, very large amplitude waves are excited, and discrete auroral arcs are formed. The breakup of these arcs is a problem in nonlinear wave interaction in which the earthward boundary conditions are crucial.

The observed phenomenology of arc breakup is that at small scales $(<10 \mathrm{~km})$, the arcs break up into curls, which are consistent with the evolution of a KelvinHelmholtz shear instability. At large scales ( $>50 \mathrm{~km}$ ), the breakup is into spirals which are wound in the opposite sense of the small-scale curls. The evolution of these spirals is also often reversible in time, i.e. the spirals alternately wind and unwind. This behavior is not observed in the small-scale curls.

The purpose of this project was to study properties of MHD turbulence with a strong background magnetic field. We computed numerical solutions of Reduced Magnetohydrodynamics (RMHD) in three dimensions with a finite difference computer algorithm. By computing both in a homogeneous domain (periodic boundary condition) and in a partially reflecting cavity representative of the magnetosphere, and by exploring a range of relative length and time scales, we have gained insights into anisotropic MHD and its implications in the above physical systems. ${ }^{15}$

We conducted a program of study of turbulent MHD by calculating numerical solutions of systems designed to model the free evolution of the solar wind and the nonlinear breakup of auroral arcs in the near-earth magnetosphere. Our results suggest explanations for the development of anisotropy in the magnetic spectrum of the solar wind and for the scale-dependent breakup phenomenology of auroral arcs. ${ }^{16} \mathrm{We}$ also made analytical calculations which give a unifying interpretation to these results. 


\section{Ground Magnetometer Studies of Magnetospheric Perturbations.}

Perturbations of the earth's surface magnetic field are an important consequence, and hence an important tool for the interpretation, of magnetospheric dynamics. Sudden impulses (SIs) are known to trigger magnetospheric substorms. Storm sudden commencements (SSCs) are known to energize geostationary particles and occasionally produce long-lived enhancements in radiation belt particles. Magnetospheric substorms cause characteristic irregular magnetic pulsations with frequencies of tens of seconds (Pi2). They are also associated with an impulsive acceleration of particles that starts from a localized sector at the nightside and spreads in both local time and latitude. LANL spacecraft are observing these processes routinely. However, modeling of the impulsive acceleration of the particles is rather rudimentary, with the exception of a few multispacecraft efforts. To increase the database of ground magnetometer studies, we have successfully carried out the installation at three sites (Los Alamos, US Airforce Academy, and San Gabriel Dam) and the fourth site will become operational shortly (Fig. 8). We have studied propagation of sudden impulses and irregular pulsations of type -2 (Pi2s) and have established our ability to detect time lags between longitudinally separated stations. We have found that such propagation delays do not always agree with expected delays, putting our theories of how such waves propagate to test.

A model of propagation of hydromagnetic waves caused by a sudden dynamic pressure increase of the solar wind was developed in the early years of magnetospheric physics. ${ }^{[16,17]}$ This model was tested against satellite and ground measurements during the IMS years for one event ${ }^{[18]}$ but little support for time delays was available from ground measurements. The time delay of such perturbations registered in the dayside and nightside is roughly $10 \mathrm{~s}^{177]}$ and thus should be measurable with a high temporal resolution network. Wilken et al. ${ }^{[18]}$ attempted to model the propagation and effects on geosynchronous particles of an SSC. 
They concluded that the propagation was consistent with the model proposed by Francis et al. ${ }^{[17]}$ but the exact numbers of speed propagation were inadequate, possibly because of the lack of good density models for the inner magnetosphere. The effects on the increased flux of the energetic particles at geosynchronous orbit were explained by the combined influence of betatron acceleration and the inward convection of lower density particles from the outer magnetosphere. No quantitative agreement was reached in those studies.

Using our newly installed magnetometer network, we have found that finite SI wave speeds can be detected from one station to the other. This is most evident when the pulse duration is short (i.e., $\sim 1 \mathrm{~min}$ ) but is masked when the rise duration is long ( $>5$ minutes). Based on the longest observed time delay, propagation speeds are estimated to be on the order of at least $800 \mathrm{~km} / \mathrm{s}$ if the wave propagates outside of $1 \mathrm{Re}$ altitude (topside ionosphere). Such speeds are lower than the Alfven speed except for the top layer ionosphere. This suggests that the waves propagate around the Earth ahead of the solar wind discontinuity that generated them.

We also studied the correlation of Pi2 waves on the UC-LANL chain stations. We selected events associated with positive excursions of the horizontal component, indicating a substorm onset, in the three month period between July 6 and October 6 , 1998. So far, we limited ourselves to the use of two stations, San Gabriel Dam, California (SGD) and Los Alamos (LAL). The resulting time lags and correlations from an analysis of $35 \mathrm{Pi} 2$ events are shown in Figure 9 as a function of time. A linear fit to the data shows a propagation velocity of $2.85 \mathrm{MLT} / \mathrm{second}$. The time lag switches sign at UT=6.31. At that time the average magnetic local time (MLT) of the stations SGD and LAL is at 2300. This is precisely where substorm onsets are generated and the Pi2 source region is expected to be. The UT of midnight for stations SGD and LAL is also shown in Table 1. The puzzle is that instead of the waves propagating away from midnight, they are propagating towards midnight. 
Moreover, the phase velocity is $1.89 \mathrm{MLT}$ hours per second (2.82 MLT hours per second if only high correlation events are included). If the signal propagates azimuthally along a surface of 2 Re radius it should travel at a speeds of $7000-10000 \mathrm{~km} / \mathrm{s}$. This can only match the Alfven velocity at the topside ionosphere. Anywhere else inside of the plasmasphere propagation speeds are much lower than that.

Our findings are in agreement with the model of generation of Pi2s proposed by Allan et al..$^{[19]}$ and extensively studied by Takahashi et al. ${ }^{[20]}$ using AMPTE/CCE spacecraft data. The latter work finds a good correlation between space and ground signals at midnight, but essentially no wave signatures in space between 2 and $6 \operatorname{Re}$ on the dayside. Since the Pi2s are indeed seen at the dayside midlatitudes, up to $\mathrm{L}$ shells of 2 , they have to propagate there from a path other than the plasmaspheric cavity. Since the ionospheric cavity is transparent to low frequency waves, the only possible cavity for propagation of those waves is the ionospheric Alfven resonator cavity between the bottomside ionosphere $(\sim 100 \mathrm{~km})$ and the altitude of the Alfven velocity maximum $(\sim 6000 \mathrm{~km})$. The complex nature of the refractive index in that region may support waves with phase and group velocity behavior like the one observed here. This supposition is currently under investigation. 


\section{Publications}

1. Birn, J., and M. Hesse, "Details of current disruption and diversion in simulations of magnetotail dynamics," J. Geophys. Res. 101, 15,345 (1996).

2. Birn, J., F. Iinoya, J. U. Brackbill, and M. Hesse, "A Comparison of MHD simulations of magnetotail dynamics," Geophys. Res. Lett. 23, 323, 1996.

3. Birn, J., M. Hesse, and K. Schindler, "MHD simulations of magnetotail dynamics," J. Geophys. Res. 101, 12,939 (1996).

4. Birn, J., M. Hesse, and K. Schindler, "Formation of thin current sheets in space plasmas," J. Geophys. Res., 103, 6843 (1998).

5. Convery, P. D., and S. P. Gary, "Electromagnetic proton cyclotron ring instability: Threshold and saturation," J. Geophys. Res., 102, 2351 (1997).

6. Gary, S. P., J. A. Newbury, and B. Goldstein, A lower bound for electron core beta in the solar wind, J. Geophys. Res., 103, 14559, 1998.

7. Gosling, J. T., M. F. Thomsen, G. Le, C. T. Russell, "Observations of magnetic reconnection at the lobe magnetopause," J. Geophys. Res., 101, 24,765-24,773, 1996.

8. Hesse, M., D. Winske, M. Kuznetsova, J. Birn, and K. Schindler, "Hybrid modeling of the formation of thin current sheets in magnetotail configurations," J. Geomagn. Geoelec., 120, 749, 1996.

9. Hesse, M., D. Winske, and J. Birn, "Hybrid modeling of the formation and structure of thin current sheets in the magnetotail," in Third International Conference on Substorms (ICS-3), ESA Publ., SP-389, edited by E. J. Rolfe and B. Kaldeich, p.231, 1996.

10. Hesse, M., et al., A simple model of core field generation during plasmoid evolution, J. Geophys. Res., 101, 10,797, 1996.

11. Hesse, M., and M.G. Kivelson, "The Formation and Structure of Flux Ropes in the Magnetotail," in New Perspectives on the Earth's Magnetotail, edited by A. Nishida, D. N. Baker, S. W. H. Cowley, American Geophysical Union, Washington DC, p. 139 (1998).

12. Karimabadi, H., H. X. Vu, K. B. Quest, and D. Krauss-Varban, Hybrid Simulations of the Magnetopause, EOS Trans., 78, 610, 1997.

13. Karimabadi, H., D. Krauss-Varban, N. Omidi, and P. Riley, Effect of shear flow on the reconnection geometry and the resulting ionospheric signatures, CA, EOS Trans., 79 (45), Suppl. 746, 1998.

14. Karimabadi, H., et al., "Magnetic Structure of the Reconnection Layer and Core Field Generation in Plasmoids," J. Geophys. Res. 104, 12313 (1998). 
15 Kinney, R.M., and J.C. Williams, "Turbulent Cascades in Anisotropic Magnetohydrodynamics," Phys. Rev. E 57, 7111 (1998).

16. Kinney, R.M., et al., "Mechanism for Discrete Arc Breakup by Nonlinear Alfven Wave Interaction," J. Geophys. Res. 104, 19,931 (1999).

17. Kivelson, M. G., K. K. Khurana, R. J. Walker, and E. L. Kepko, "Flux ropes, interhemispheric conjugacy, and magnetospheric current closure," J. Geophys. Res. 101, 27,341 (1996).

18.Krauss-Varban, D., N. Omidi, and H. Karimabadi, Modeling magnetosphereionosphere coupling with hybrid simulations, EOS Trans., 79 (17), 304 (1998).

19.Krauss-Varban, D., et al., "Two-Dimensional Structure of the Coplanar and Noncoplanar Magnetopause Transition During Reconnection," Geophys. Res. Lett. 26, 1235 (1999).

20. Le, G., C. T. Russell, J. T. Gosling, and M. F. Thomsen, "ISEE Observations of Low Latitude Boundary Layer for Northward Interplanetary magnetic field: Implications for cusp Reconnection," J. Geophys. Res., 101, 27,239 (1996).

21.Le, G., et al., "The Magnetic and Plasma Structure of Flux Transfer Events," J. Geophys. Res. 104, 233 (1999).

22.McKean, M. E., N. Omidi, and D. Krauss-Varban, "Magnetosheath Dynamics Downstream of Low Mach Number Shocks," J. Geophys. Res. 101, 20,013, (1996).

23. Newbury, J. A., Rule of thumb: Electron temperatures in the solar wind at $1 \mathrm{AU}$, Eos, 77, 471, 1996.

24. Newbury, J.A., et al., "Electron Temperatures in the Solar Wind: Typical Properties and a Lower Bound at 1 AU," J. Geophys. Res. 103, 9553 (1998).

25. Omidi, N., et al., "Hybrid Simulation of the Curved Dayside Magnetopause During Southward IMF," Geophys. Res. Lett. 25, 3273 (1998).

26.Russell, C. T., et al., "Intrinsic Time Scale for Reconnection on the Dayside Magnetopause," Adv. Space Res., 19(12), 1913 (1997).

27.Winske, D., "Regimes of the Magnetized Rayleigh-Taylor Instability," Phys. Plasmas, 3, 3966 (1996).

28. Winske, D., "The magnetized Rayleigh-Taylor Instability With Temporally Variable Gravity," Phys. Plasmas, 4, 2454 (1997). 


\section{References}

[1] Montgomery, M. D., Average thermal characteristics of solar wind, in Solar Wind, edited by C. P. Sonett, P. J. Coleman and J. M. Wilcox, p. 208, NASA, Pacific Grove, CA, 1971.

[2] Feldman, W. C., J. R. Asbridge, S. J. Bame and J. T. Gosling, Plasma and magnetic fields from the sun, in The Solar Output and Its Variation, edited by O. R. White, pp. 351, Colorado University Press, Boulder, CO, 1977.

[3] Schwartz, S. J., Plasma instabilities in the solar wind: A theoretical review, Rev. of Geophys. and Space Phys., 313, 1980.

[4] Gary, S. P., Theory of Space Plasma Microinstabilities, New York: Cambridge University Press, 1993.

[5] Daly, P.W., and E. Keppler, Observation of a flux transfer event on the Earthward side of the magnetopause, Planet. Space Sci., 30, 331, 1982.

[6] Daly, P. W., D. J. Williams, C. T. Russell, and E. Keppler, Particle signature of magnetic flux transfer events at the magnetopause, J. Geophys. Res., 86, 1628, 1981.

[7] Daly, P. W., M. A. Saunders, R. P. Rijnbeek, N. Sckopke, and C. T. Russell, The distribution of reconnection geometry in flux transfer events using energetic ion, plasma and magnetic data, J. Geophys. Res., 89, 3843, 1984.

[8] Gosling, J. T., M. F. Thomsen, S. J. Bame, T. G. Onsager, and C. T. Russell, The electron edge of the low latitude boundary layer during accelerated flow events, Geophys. Res. Lett., 17, 1833, 1990.

[9] Paschmann, G. Haerendel, I. Papamastorakis, N. Sckopke, S. J. Bame, J. T. Gosling, and C. T. Russell, Plasma and magnetic field characteristics of magnetic flux transfer events, J. Geophys. Res., 87, 2159, 1982.

[10] Scholer, M., D. Hovestadt, F. M. Ipavich, and G. Gloeckler, Energetic protons, Alpha particles, and electrons in magnetic flux transfer events, J. Geophys. Res., 87, $2169,1982$.

[11] Southwood., D. J., C. J. Farrugia, and M. A. Saunders, What are flux transfer events?, Planet. Space Sci., 36, 503, 1988.

[12] Thomsen, M. F., J. A. Stansberry, S. J. Bame, S. A. Fuselier, and J. T. Gosling, Ion and electron velocity distributions within flux transfer events, J. Geophys. Res., 92, 12,127, 1987.

[13] Kivelson, M. G., and K. K. Khurana, "Models of flux ropes embedded in a Harris neutral sheet: Force-free solutions in low and high beta plasmas," J. Geophys. Res. 100, 23,637 (1995).

[14] Russell, C. T., and R. C. Elphic, ISEE observations of flux transfer events at the dayside magnetopause, Geophys. Res. Lett., 6, 33, 1979. 
[15] Angelopoulos, V., V. A. Sergeev, D. N. Baker, D. G. Mitchell, G. D. Reeves, C. T. Russell, and H. J. Singer, Mulit-point study of bursty bulk flow events during a sequence of small substorms, in Proceedings of ICS2, Fairbanks, Alaska, p. 643, 1994.

[16] Araki, T., Global structure of geomagnetic sudden commencements, Planet. Space Sci., 25, 373, 1977.

[17] Francis, W. E., M. J. Green and A. J. Dessler, Hydromagnetic propagation of sudden commencements of magnetic storms, J. Geophys. Res., 64, 1643, 1959.

[18] Wilken, B., C. K. Goertz, D. N. Baker, P. R. Higbie, and T. A. Fritz, The SSC on July 29, 1977 and its propagation within the magnetosphere, J. Geophys. Res., 87, $5901,1982$.

[19] Allan, W., E. M. Poulter, and S. P. White, Hydromagnetic wave coupling in the magnetosphere - Plasmapause effects on impulse-excited resonances, Planet. Space Sci., 34, 1189, 1986.

[20] Takahashi, K., S. -I. Ohtani, and B. J. Anderson, Statistical analysis of Pi2 pulsations observed by the AMPTE/CCE spacecraft in the inner magnetosphere, J. Geophys. Res., 100, 21929, 1995.

Enter any figure captions here

Figure 1: Magnetic structure and characteristic regions of the earth's magnetosphere. Solid lines indicated magnetic field lines in the solar wind (green) and the magnetosphere(blue). Red arrows indicate the direction of plasma flow.

Figure 2. Simulation of a curved bow shock in front of an obstacle in the solar wind, streaming toward the right. The color shows the intensity of the y component of the magnetic field. The shock is clearly seen, but the magnetopause boundary is less obvious from this plot. The straight line is the "Cut-Line".

Figure 3. Top to bottom: Magnetic field, density, temperature, and flow velocity along the $x$ axis ("Cut-Line" in Fig. 2). The magnetopause boundary is most obvious from the density plot, although one can also see the magnetic field increases. There is not much effect on the temperature or flow velocity.

Figure 4. Results of simulation of Kelvin-Helmholtz instability at a magnetized plasma interface. The top panels show, by color intensity, the magnitude of the magnetic field strength at two different times, and the bottom panel shows a surface plot of the magnetic field at the later time. 
Figure 5. Snapshots of ion and electron distribution functions observed near the September 11, 1979 magnetopause crossing. The measurements are taken in the magnetosphere (a), the magnetosheath (b), and within the flux transfer event (c and d).

Figure 6. Magnetic field data from the ISEE 1 and 2 spacecraft and the inferred trajectory through the model flux rope.

Figure 7. Magnetic field variations associated with a flux rope in the magnetotail plasma sheet that is not in force balance. Note the asymmetry in $\mathrm{Bz}$, with the trailing edge smaller in size (time) and exhibiting a much larger perturbation than the leading edge.

Figure 8. Locations of the Los Alamos/UCLA mid-latitude magnetometer chain. The purpose is to study the propagation of magnetic perturbations associated with dynamic processes in the earth's magnetosphere. SGA: San Gabriel Dam, California; LAL: Los Alamos, New Mexico; AFA: US Air Force Academy, Colorado Springs, Colorado; SLU: Southeastern Louisiana University, Livingston Parish, Louisiana.

Figure 9. Summary of analysis of $35 \mathrm{Pi} 2$ pulsations. Top shows the correlation and bottom shows time lags observed, both plotted as a function of the center time of the Pi2 observation. A positive time lag indicates a westward phase velocity. 


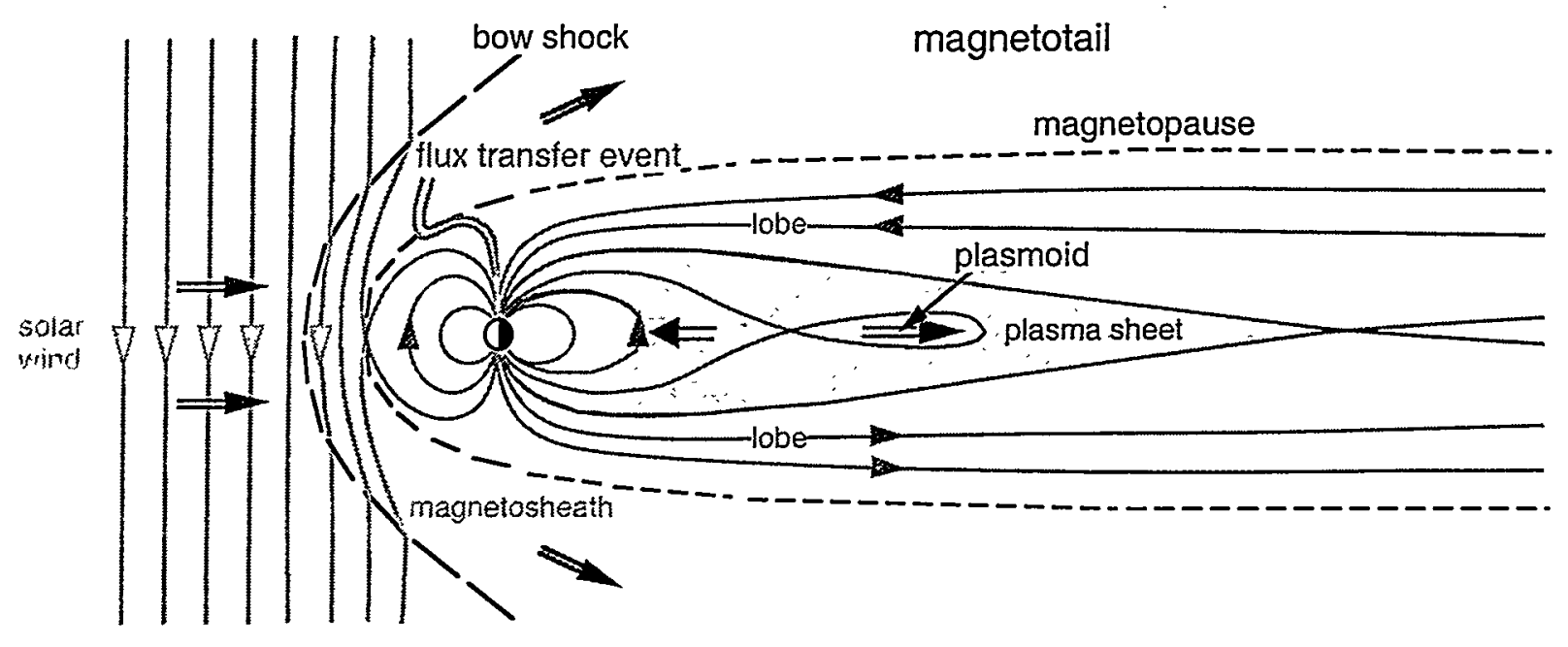




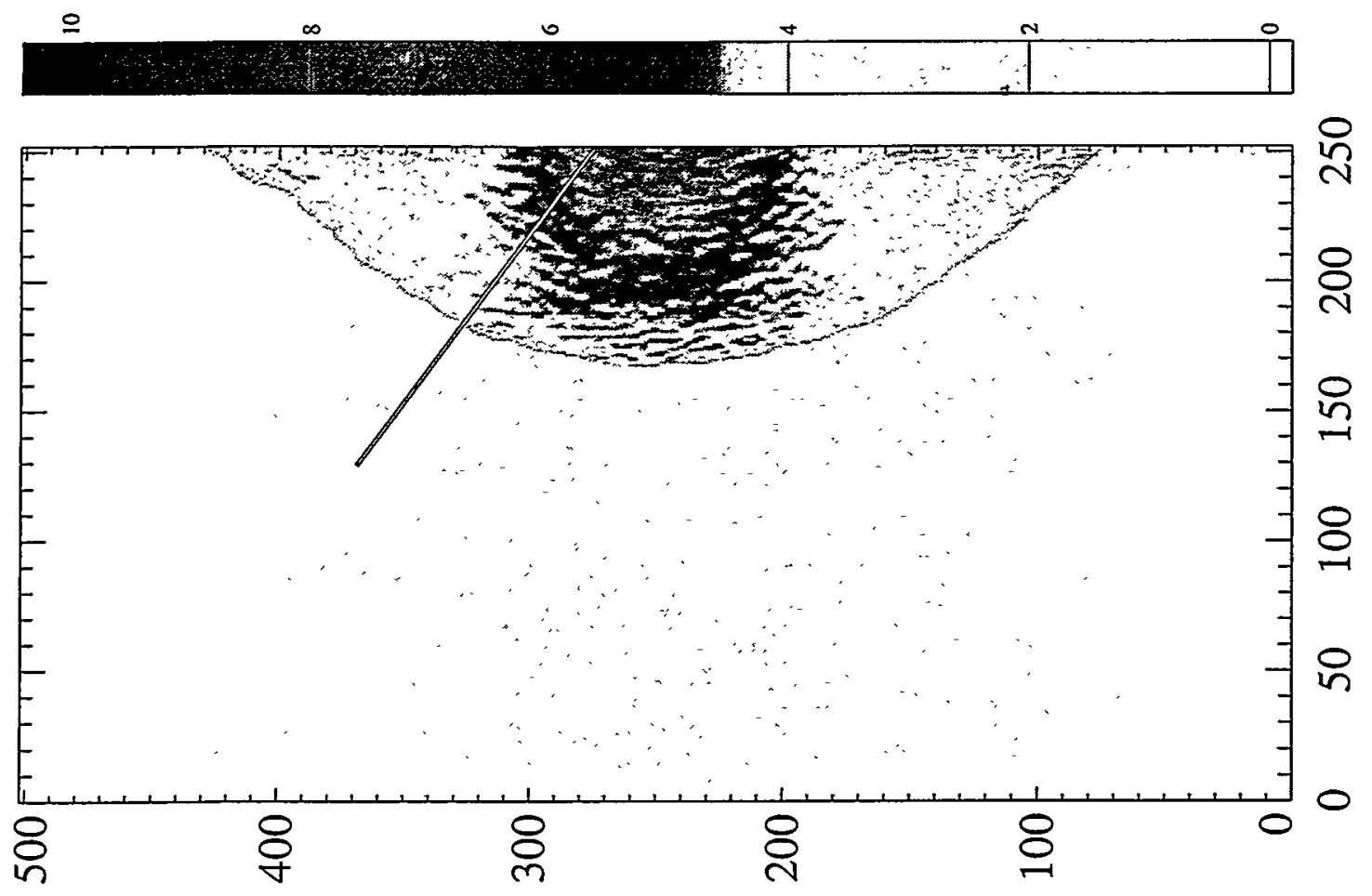



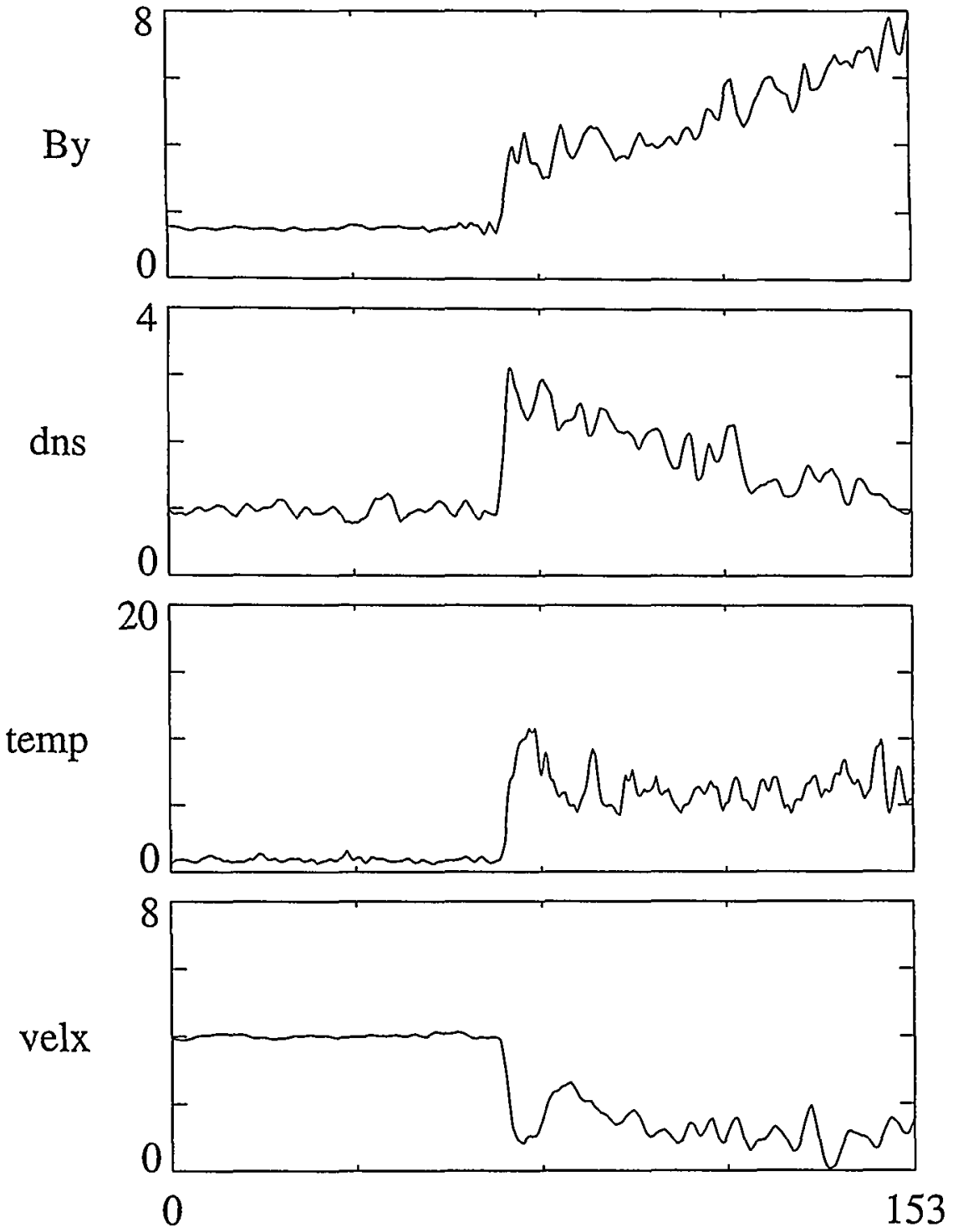


\section{Parallel current \& ionospheric signatures at $\Omega t=100$}

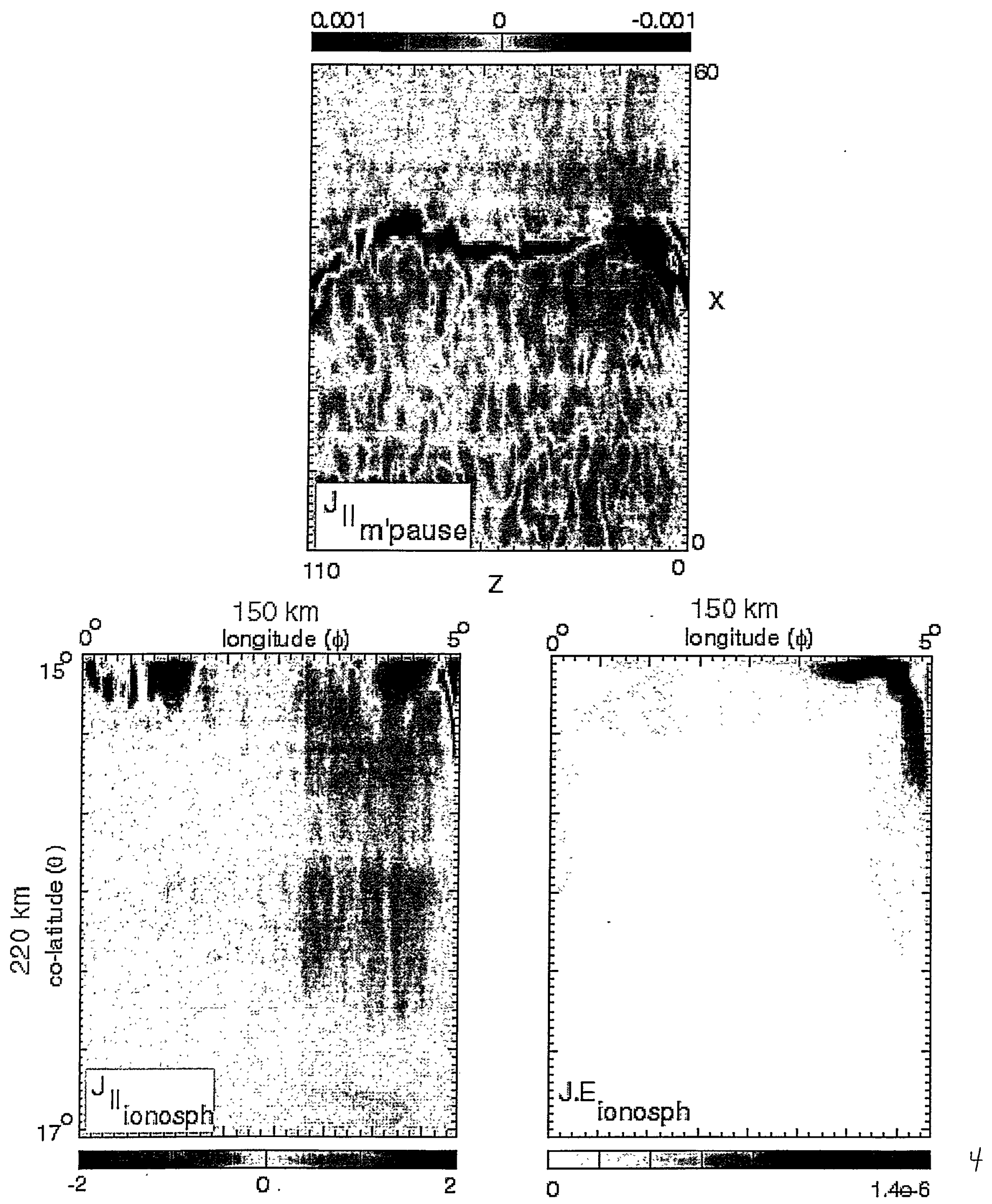


iSEE 2 Fast Plasma Experiment (FPE)

Log Distribution Function, September 11, 1979

Magnetosphere Magnetosheath Flux Transfer Event

$\begin{array}{llll}\text { (a) 0405:28 - :31 UT (b) 0424:04-:07 UT (c) 0429:04-:07 UT (d) 0432:16-:19 UT } & \text { (b) }\end{array}$
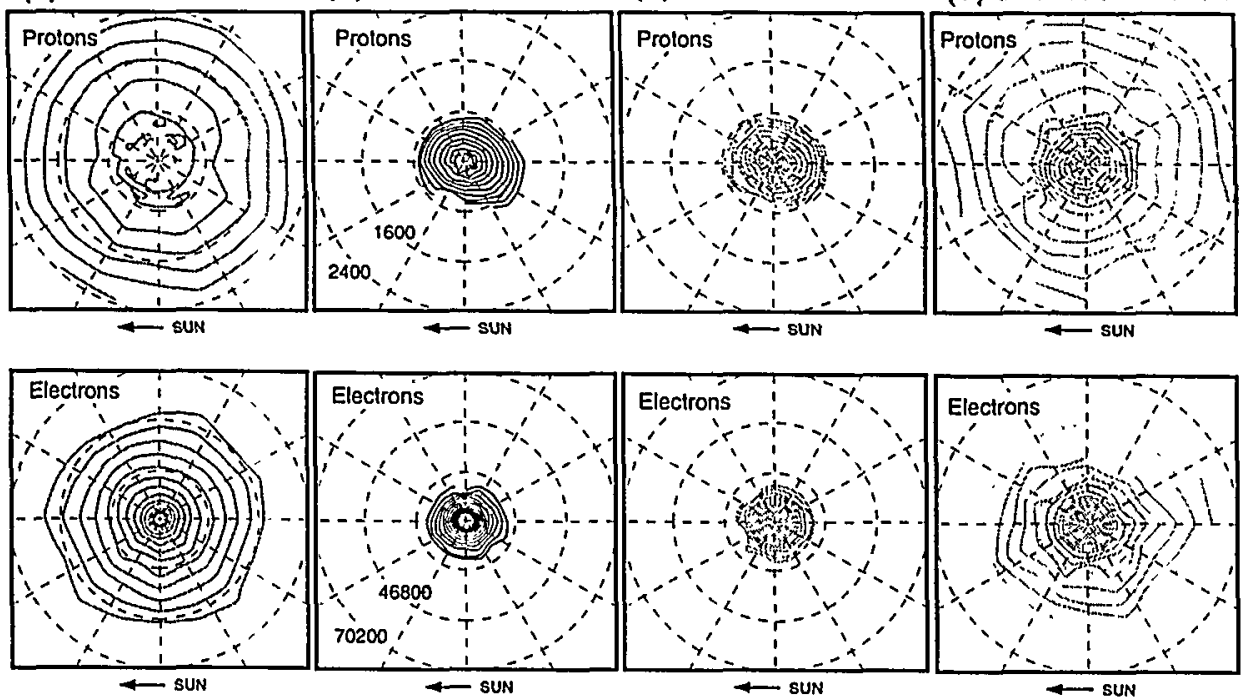

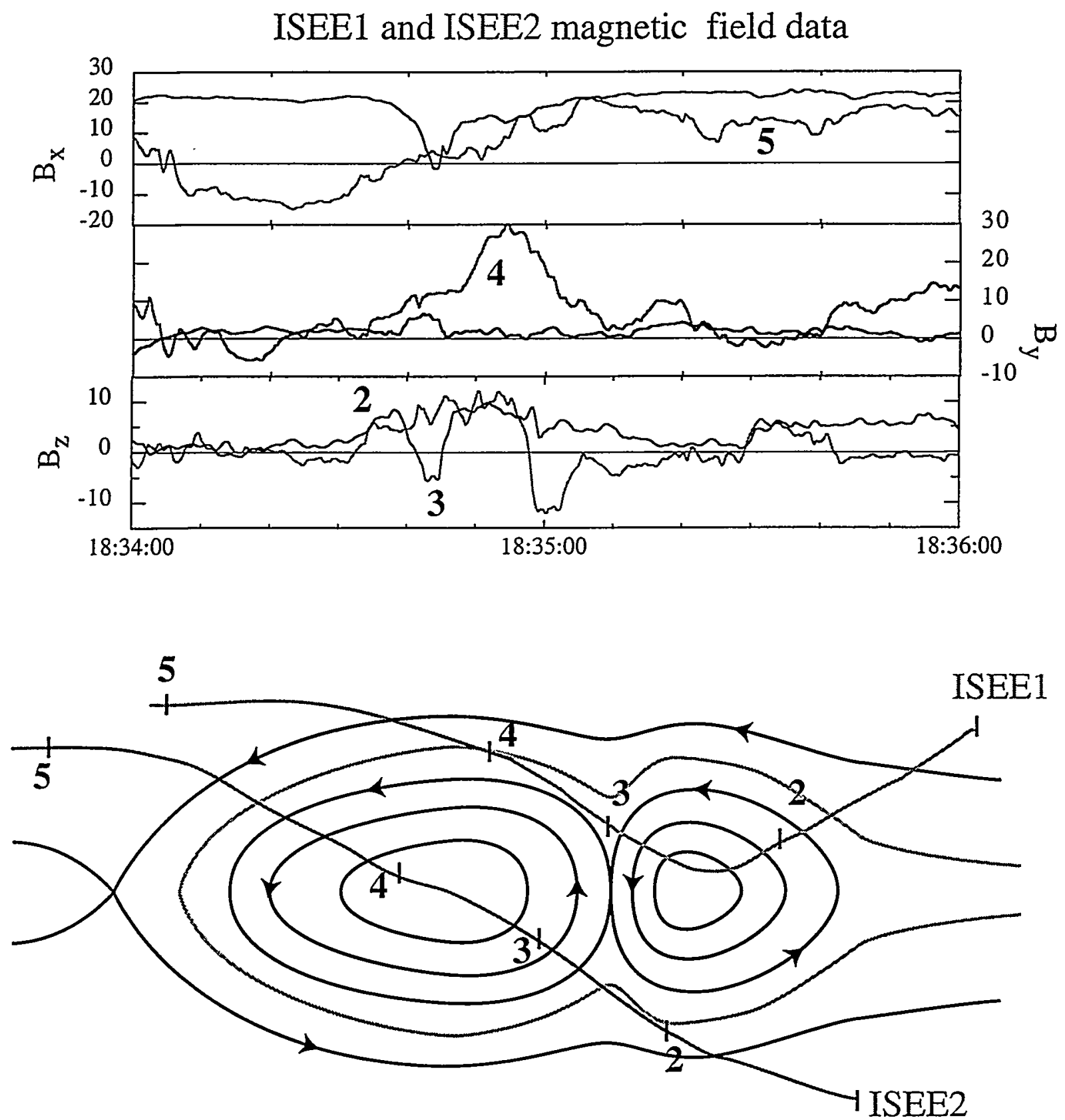
1994-Jan-12
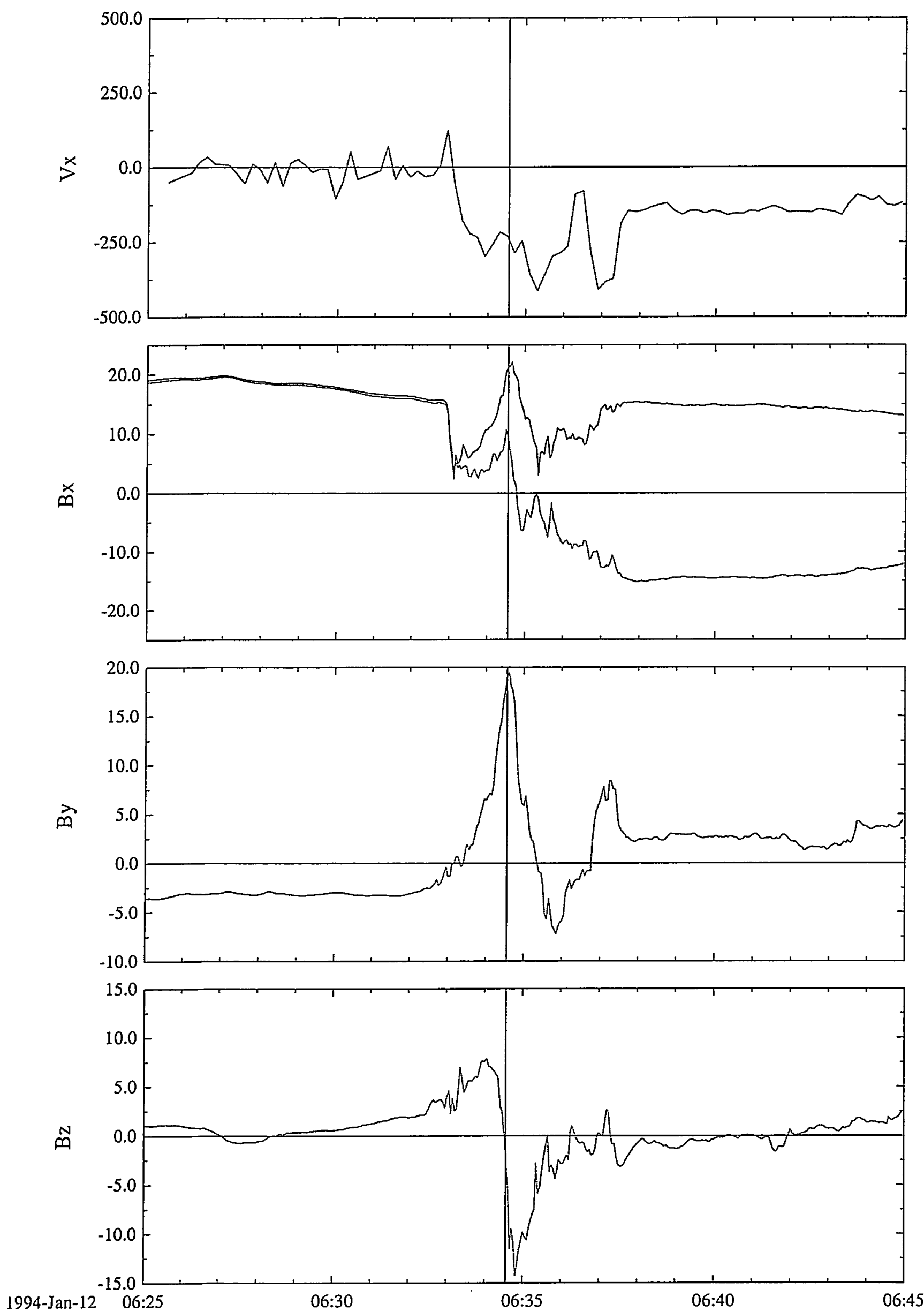


\section{UC-LANL Magnetometer Network}

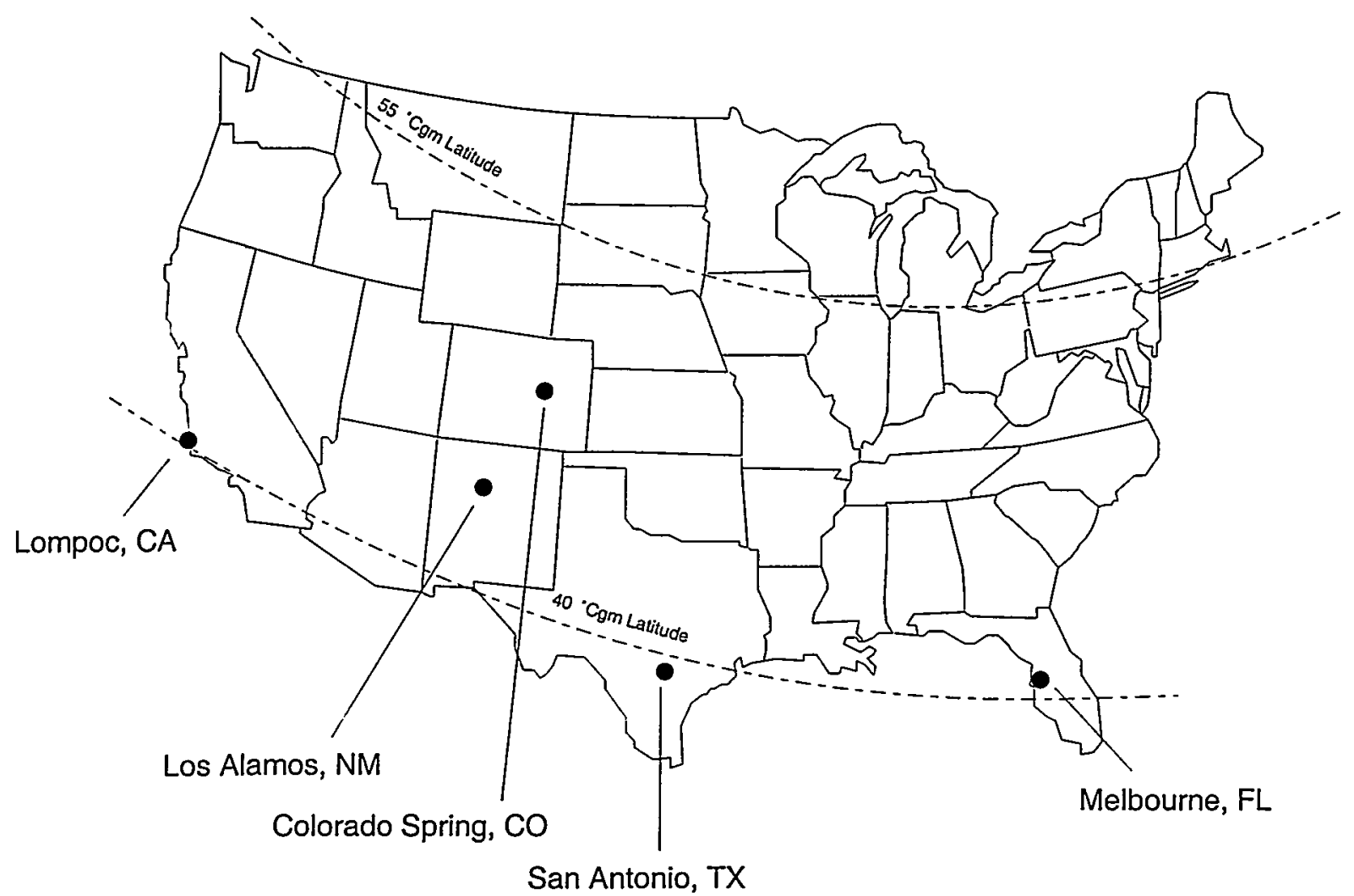



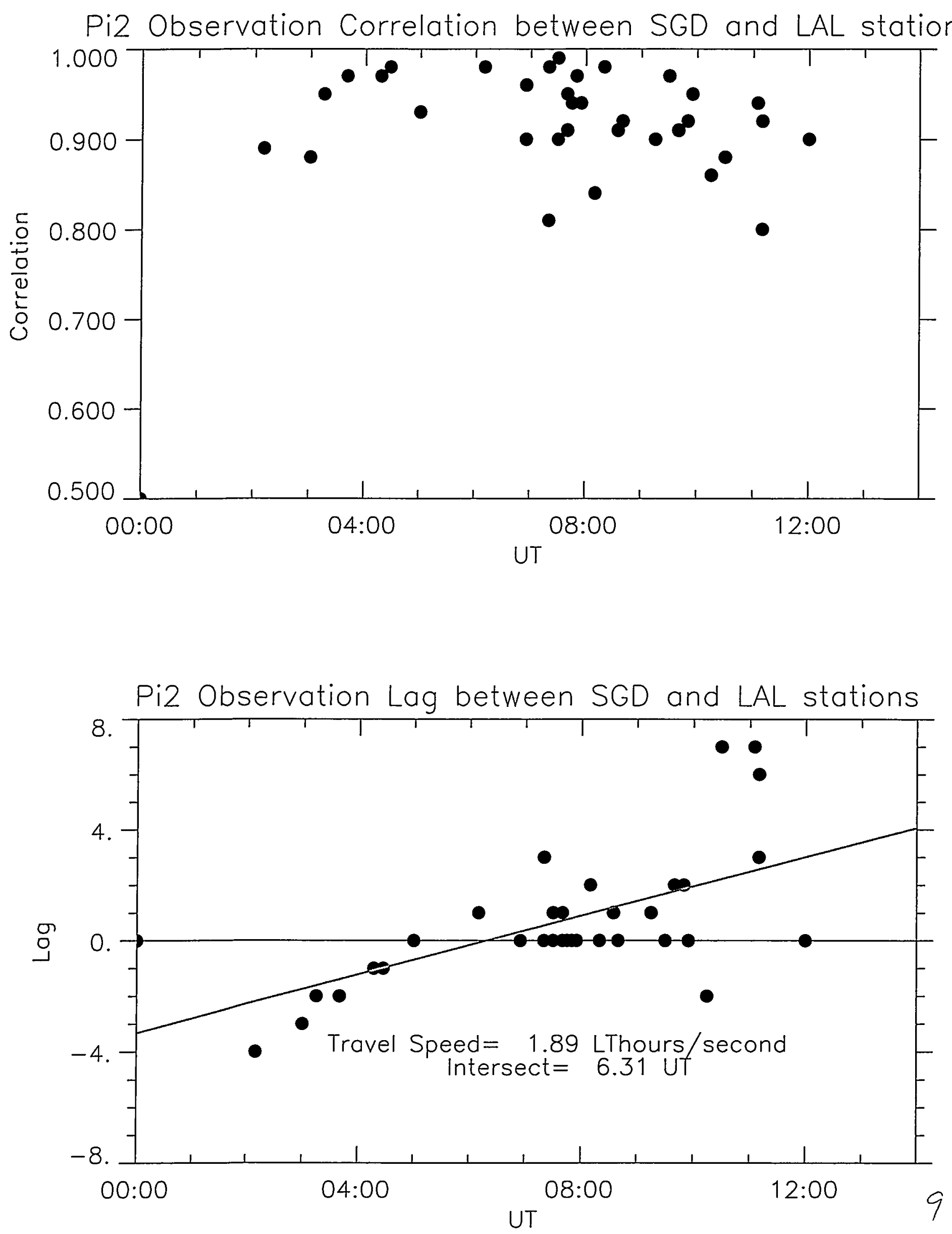